\title{
A Class of Numerical Methods for the Solution of Fourth-Order Ordinary Differential Equations in Polar Coordinates
}

\author{
Jyoti Talwar and R. K. Mohanty \\ Department of Mathematics, Faculty of Mathematical Sciences, University of Delhi, 110007 Delhi, India
}

Correspondence should be addressed to R. K. Mohanty, rmohanty@maths.du.ac.in

Received 16 August 2011; Revised 11 January 2012; Accepted 18 January 2012

Academic Editor: Alfredo Bermudez De Castro

Copyright (C) 2012 J. Talwar and R. K. Mohanty. This is an open access article distributed under the Creative Commons Attribution License, which permits unrestricted use, distribution, and reproduction in any medium, provided the original work is properly cited.

In this piece of work using only three grid points, we propose two sets of numerical methods in a coupled manner for the solution of fourth-order ordinary differential equation $u^{i v}(x)=$ $f\left(x, u(x), u^{\prime}(x), u^{\prime \prime}(x), u^{\prime \prime \prime}(x)\right), a<x<b$, subject to boundary conditions $u(a)=A_{0}, u^{\prime}(a)=A_{1}$, $u(b)=B_{0}$, and $u^{\prime}(b)=B_{1}$, where $A_{0}, A_{1}, B_{0}$, and $B_{1}$ are real constants. We do not require to discretize the boundary conditions. The derivative of the solution is obtained as a byproduct of the discretization procedure. We use block iterative method and tridiagonal solver to obtain the solution in both cases. Convergence analysis is discussed and numerical results are provided to show the accuracy and usefulness of the proposed methods.

\section{Introduction}

Consider the fourth-order boundary value problem

$$
u^{i v}(x)=f\left(x, u(x), u^{\prime}(x), u^{\prime \prime}(x), u^{\prime \prime \prime}(x)\right), \quad a<x<b,
$$

subject to the prescribed natural boundary conditions

$$
u(0)=A_{0}, \quad u^{\prime}(0)=A_{1}, \quad u(1)=B_{0}, \quad u^{\prime}(1)=B_{1},
$$

or equivalently, for $u^{\prime}(x)=v(x)$,

$$
u^{i v}(x)=f\left(x, u(x), v(x), u^{\prime \prime}(x), v^{\prime \prime}(x)\right), \quad a<x<b,
$$


subject to the natural boundary conditions

$$
u(0)=A_{0}, \quad v(0)=A_{1}, \quad u(1)=B_{0}, \quad v(1)=B_{1},
$$

where $A_{0}, A_{1}, B_{0}$, and $B_{1}$ are real constants and $-\infty<a \leq x \leq b<\infty$.

Fourth-order differential equations occur in a number of areas of applied mathematics, such as in beam theory, viscoelastic and inelastic flows, and electric circuits. Some of them describe certain phenomena related to the theory of elastic stability. A classical fourth-order equation arising in the beam-column theory is the following (see Timoshenko [1]):

$$
E I \frac{d^{4} u}{d x^{4}}+P \frac{d^{2} u}{d x^{2}}=q
$$

where $u$ is the lateral deflection, $q$ is the intensity of a distributed lateral load, $P$ is the axial compressive force applied to the beam, and EI represents the flexural rigidity in the plane of bending. Various generalizations of the equation describing the deformation of an elastic beam with different types of two-point boundary conditions have been extensively studied via a broad range of methods.

The existence and uniqueness of solutions of boundary value problems are discussed in the papers and book of Agarwal and Krishnamoorthy, Agarwal and Akrivis (see [2-5]). Several authors have investigated solving fourth-order boundary value problem by some numerical techniques, which include the cubic spline method, Ritz method, finite difference method, multiderivative methods, and finite element methods (see [6-16]). In the 1980s, Usmani et al. (see [17-19]) worked on finite difference methods for solving $\left[p(x) y^{\prime \prime}\right]^{\prime \prime}+$ $q(x) y=r(x)$ and finite difference methods for computing eigenvalues of fourth-order linear boundary value problem. In 1984, Twizell and Tirmizi (see [20]) developed multi-derivative methods for linear fourth-order boundary value problems. In 1984, Agarwal and Chow (see [21]) developed iterative methods for a fourth-order boundary value problem. In 1991, O'Regan (see [13]) worked on the solvability of some fourth-(and higher) order singular boundary value problems. In 1994, Cabada (see [22]) developed the method of lower and upper solutions for fourth- and higher-order boundary value problems. In 2005 Franco et al. (see [23]) dealt with some fourth-order problems with nonlinear boundary conditions. In 2006, Noor and Mohyud-Din (see [12]) used the variational iteration method to solve fourthorder boundary value problems and further developed the homotopy perturbation method for solving fourth order boundary value problems. Some of these methods use transformation in order to reduce the equation into more simple equation or system of equations and some other methods give the solution in a series form which converges to the exact solution. Later, Han and Li (see [24]) worked on some fourth-order boundary value problems.

In this paper we present the finite difference methods for the solution of fourth-order boundary value problem. We discretize the interval $[a, b]$ into $N+1$ subintervals each of width $h=(b-a) /(N+1)$, where $N$ is a positive integer. We seek the solution of (la) or (2a) at the grid points, $x_{k}=k h, k=1,2, \ldots, N$. Let $u_{k}$ and $u_{k}^{\prime}$ denote the approximate solutions, and let $U_{k}=u\left(x_{k}\right)$ and $U_{k}^{\prime}=u^{\prime}\left(x_{k}\right)$ be the exact solution values of $u(x)$ and $u^{\prime}(x)$ at the grid point $x=x_{k}$, respectively. Also, $x_{0}=a$ and $x_{N+1}=b$.

Using the second-order central differences, we obtain a five-point difference formula for (1.1), which requires the use of fictitious points outside $[a, b]$. The accuracy of the numerical solution depends upon the boundary approximation used. The finite difference 
method discussed here is based only on three grid points for second-and fourth-order methods. Therefore, no fictitious points are required for incorporating the boundary conditions. Here, we use a combination of the value of the solution $u(x)$ and its derivative $u^{\prime}(x)$ to derive the difference scheme using three grid points.

Since we need to solve a coupled system of equations at each mesh point, the block successive overrelaxation (BSOR) iterative method is used.

\section{The Finite Difference Method}

The method is described as follows: for $k=1(1) N$, let

$$
\begin{gathered}
\bar{u}_{k}^{\prime \prime}=\frac{\left(u_{k+1}^{\prime}-u_{k-1}^{\prime}\right)}{2 h}, \\
\bar{u}_{k}^{\prime \prime \prime}=\frac{\left(u_{k+1}^{\prime}-2 u_{k}^{\prime}+u_{k-1}^{\prime}\right)}{h^{2}}, \\
\bar{f}_{k}=f\left(x_{k}, u_{k}, u_{k}^{\prime}, \bar{u}_{k}^{\prime \prime}, \bar{u}_{k}^{\prime \prime \prime}\right) .
\end{gathered}
$$

Then, the difference method of order two for the given differential (1.1) is given by,

$$
-2\left(u_{k+1}-2 u_{k}+u_{k-1}\right)+h\left(u_{k+1}^{\prime}-u_{k-1}^{\prime}\right)=\frac{h^{4}}{6} \bar{f}_{k^{\prime}}
$$

and the corresponding difference method for the derivative $u^{\prime}(x)$ is given by

$$
-3\left(u_{k+1}-u_{k-1}\right)+h\left(u_{k+1}^{\prime}+4 u_{k}^{\prime}+u_{k-1}^{\prime}\right)=0 .
$$

Also, let

$$
\begin{gathered}
\bar{u}_{k \pm 1}^{\prime \prime}=\frac{\left( \pm 3 u_{k \pm 1}^{\prime} \mp 4 u_{k}^{\prime} \pm u_{k \mp 1}^{\prime}\right)}{2 h}, \\
\overline{\bar{u}}_{k}^{\prime \prime \prime}=\frac{15\left(u_{k+1}-u_{k-1}\right)}{2 h^{3}}-\frac{3\left(u_{k+1}^{\prime}+8 u_{k}^{\prime}+u_{k-1}^{\prime}\right)}{2 h^{2}}, \\
\overline{\bar{u}}_{k \pm 1}^{\prime \prime}=\frac{-\left(11 u_{k \pm 1}-16 u_{k}+5 u_{k \mp 1}\right)}{2 h^{2}} \pm \frac{\left(4 u_{k \pm 1}^{\prime}-u_{k \mp 1}^{\prime}\right)}{h}, \\
\overline{\bar{u}}_{k}^{\prime \prime}=\frac{2\left(u_{k+1}-2 u_{k}+u_{k-1}\right)}{h^{2}}-\frac{\left(u_{k+1}^{\prime}-u_{k-1}^{\prime}\right)}{2 h}, \\
\bar{u}_{k \pm 1}^{\prime \prime \prime}=\mp \frac{\left(27 u_{k \pm 1}-48 u_{k}+21 u_{k \mp 1}\right)}{2 h^{3}}+\frac{\left(15 u_{k \pm 1}^{\prime}-9 u_{k \mp 1}^{\prime}\right)}{2 h^{2}}, \\
\overline{\bar{u}}_{k \pm 1}^{\prime \prime \prime}=\mp \frac{\left(99 u_{k \pm 1}-48 u_{k}-51 u_{k \mp 1}\right)}{2 h^{3}}+\frac{\left(39 u_{k \pm 1}^{\prime}+96 u_{k}^{\prime}+15 u_{k \mp 1}^{\prime}\right)}{2 h^{2}},
\end{gathered}
$$


and set

$$
\begin{gathered}
\bar{f}_{k \pm 1}=f\left(x_{k \pm 1}, u_{k \pm 1}, u_{k \pm 1}^{\prime}, \bar{u}_{k \pm 1}^{\prime \prime}, \bar{u}_{k \pm 1}^{\prime \prime \prime}\right), \\
\overline{\bar{f}}_{k \pm 1}=f\left(x_{k \pm 1}, u_{k \pm 1}, u_{k \pm 1}^{\prime}, \overline{\bar{u}}_{k \pm 1}^{\prime \prime}, \overline{\bar{u}}_{k \pm 1}^{\prime \prime \prime}\right), \\
\overline{\bar{f}}_{k}=f\left(x_{k}, u_{k}, u_{k}^{\prime}, \overline{\bar{u}}_{k}^{\prime \prime}, \overline{\bar{u}}_{k}^{\prime \prime \prime}\right) .
\end{gathered}
$$

Then, the difference method of order four for the differential equation and the corresponding difference method for the derivative $u_{k}^{\prime}$ are given by

$$
\begin{gathered}
-2\left(u_{k+1}-2 u_{k}+u_{k-1}\right)+h\left(u_{k+1}^{\prime}-u_{k-1}^{\prime}\right)=\frac{h^{4}}{90}\left(\overline{\bar{f}}_{k+1}+\overline{\bar{f}}_{k-1}+13 \overline{\bar{f}}_{k}\right), \\
-3\left(u_{k+1}-u_{k-1}\right)+h\left(u_{k+1}^{\prime}+4 u_{k}^{\prime}+u_{k-1}^{\prime}\right)=\frac{h^{4}}{60}\left(\bar{f}_{k+1}-\bar{f}_{k-1}\right) .
\end{gathered}
$$

Note that $u_{0}, u_{0}^{\prime}, u_{N+1}$ and $u_{N+1}^{\prime}$, are prescribed. It is convenient to express the above finite difference schemes in block tridiagonal matrix form. If the differential equation is linear, the resulting block tridiagonal linear system can be solved using the block Gauss-Seidel (BGS) iterative method. If the differential equation is nonlinear, the system can be solved using the Newton nonlinear block successive overrelaxation (NBSOR) method (see $[25,26]$ ).

\section{Derivation of the Difference Scheme}

For the derivation of the method we follow the approaches given by Chawla [27] and Mohanty [10]. In this section we discuss the derivation of the difference methods and the block iterative methods.

At the grid point $x_{k}$, the given differential equation can be written as

$$
u_{k}^{i v}=f\left(x_{k}, u_{k}, u_{k}^{\prime}, u_{k}^{\prime \prime}, u_{k}^{\prime \prime \prime}\right)=f_{k}, \quad k=1(1) N
$$

Similarly,

$$
f_{k \pm 1}=f\left(x_{k \pm 1}, u_{k \pm 1}, u_{k \pm 1}^{\prime}, u_{k \pm 1}^{\prime \prime}, u_{k \pm 1}^{\prime \prime \prime}\right), \quad k=1(1) N
$$

Using the Taylor expansion about the grid point $x_{k}$, we first obtain

$$
-2\left(u_{k+1}-2 u_{k}+u_{k-1}\right)+h\left(u_{k+1}^{\prime}-u_{k-1}^{\prime}\right)=\frac{h^{4}}{90}\left(f_{k+1}+f_{k-1}+13 f_{k}\right)+T_{1}
$$

where $T_{1}=O\left(h^{8}\right)$. 
Now, we need the $O\left(h^{2}\right)$ approximation for $u_{k \pm 1}^{\prime \prime \prime}$. Let

$$
\bar{u}_{k+1}^{\prime \prime \prime}=\frac{1}{h^{3}}\left[a_{10} u_{k}+a_{11} u_{k+1}+a_{12} u_{k-1}\right]+\frac{1}{h^{2}}\left[b_{10} u_{k}^{\prime}+b_{11} u_{k+1}^{\prime}+b_{12} u_{k-1}^{\prime}\right]
$$

Expanding each term on the right-hand side of (3.3) in the Taylor series about the point $x_{k}$ and equating the coefficients of $h^{p}(p=-3,-2,-1,0$, and 1$)$ to zero, we get

$$
\left(a_{10}, a_{11}, a_{12}, b_{10}, b_{11}, b_{12}\right)=\left(24, \frac{-27}{2}, \frac{-21}{2}, 0, \frac{15}{2}, \frac{-9}{2}\right)
$$

Thus, we obtain

$$
\begin{aligned}
\bar{u}_{k+1}^{\prime \prime \prime} & =-\frac{1}{2 h^{3}}\left[27 u_{k+1}-48 u_{k}+21 u_{k-1}\right]+\frac{1}{2 h^{2}}\left[15 u_{k+1}^{\prime}-9 u_{k-1}^{\prime}\right] \\
& =u_{k+1}^{\prime \prime \prime}-\frac{2 h^{2}}{5} u_{k}^{v}+O\left(h^{3}\right)
\end{aligned}
$$

Similarly, we obtain

$$
\begin{aligned}
\bar{u}_{k-1}^{\prime \prime \prime} & =\frac{1}{2 h^{3}}\left[27 u_{k-1}-48 u_{k}+21 u_{k+1}\right]+\frac{1}{2 h^{2}}\left[15 u_{k-1}^{\prime}-9 u_{k+1}^{\prime}\right] \\
& =u_{k-1}^{\prime \prime \prime}-\frac{2 h^{2}}{5} u_{k}^{v}-O\left(h^{3}\right) .
\end{aligned}
$$

Further, from (2.3) we obtain:

$$
\bar{u}_{k \pm 1}^{\prime \prime}=u_{k \pm 1}^{\prime \prime}-\frac{h^{2}}{3} u_{k}^{i v} \pm O\left(h^{3}\right)
$$

Hence, we can verify that $\bar{f}_{k \pm 1}=f_{k \pm 1}+O\left(h^{2}\right)$.

Next, we obtain the $O\left(h^{4}\right)$ approximation for $u_{k}^{\prime \prime}$. Let

$$
\overline{\bar{u}}_{k}^{\prime \prime}=\frac{1}{h^{2}}\left[a_{20} u_{k}+a_{21} u_{k+1}+a_{22} u_{k-1}\right]+\frac{1}{h}\left[b_{20} u_{k}^{\prime}+b_{21} u_{k+1}^{\prime}+b_{22} u_{k-1}^{\prime}\right]
$$

Using the Taylor series expansion and equating the coefficients of $h^{p}(p=-2,-1,0,1,2$, and 3) to zero, we get

$$
\left(a_{20}, a_{21}, a_{22}, b_{20}, b_{21}, b_{22}\right)=\left(-4,2,2,0, \frac{-1}{2}, \frac{1}{2}\right)
$$


Therefore,

$$
\overline{\bar{u}}_{k}^{\prime \prime}=\frac{2}{h^{2}}\left[u_{k+1}-2 u_{k}+u_{k-1}\right]-\frac{1}{2 h}\left[u_{k+1}^{\prime}-u_{k-1}^{\prime}\right]=u_{k}^{\prime \prime}+O\left(h^{4}\right)
$$

Similarly,

$$
\begin{gathered}
\overline{\bar{u}}_{k}^{\prime \prime \prime}=\frac{15}{2 h^{3}}\left[u_{k+1}-u_{k-1}\right]-\frac{3}{2 h^{2}}\left[u_{k+1}^{\prime}+8 u_{k}^{\prime}+u_{k-1}^{\prime}\right]=u_{k}^{\prime \prime \prime}+O\left(h^{4}\right) \\
\overline{\bar{u}}_{k \pm 1}^{\prime \prime}=\frac{-1}{2 h^{2}}\left[11 u_{k \pm 1}-16 u_{k}+5 u_{k \mp 1}\right] \pm \frac{1}{h}\left[4 u_{k \pm 1}^{\prime}-u_{k \mp 1}^{\prime}\right]=u_{k \pm 1}^{\prime \prime} \mp \frac{h^{3}}{15} u_{k}^{v}+O\left(h^{4}\right) .
\end{gathered}
$$

Next, we obtain the $O\left(h^{3}\right)$ approximation for $u_{k+1}^{\prime \prime \prime}$. Let

$$
\overline{\bar{u}}_{k+1}^{\prime \prime \prime}=\frac{1}{h^{3}}\left[a_{30} u_{k}+a_{31} u_{k+1}+a_{32} u_{k-1}\right]+\frac{1}{h^{2}}\left[b_{30} u_{k}^{\prime}+b_{31} u_{k+1}^{\prime}+b_{32} u_{k-1}^{\prime}\right]
$$

Equating the coefficients of $h^{p}(p=-3,-2,-1,0,1$, and 2$)$ to zero, we get

$$
\left(a_{30}, a_{31}, a_{32}, b_{30}, b_{31}, b_{32}\right)=\left(24, \frac{-99}{2}, \frac{51}{2}, 48, \frac{39}{2}, \frac{15}{2}\right)
$$

Thus, we obtain

$$
\begin{aligned}
\overline{\bar{u}}_{k+1}^{\prime \prime \prime} & =\frac{-1}{2 h^{3}}\left[99 u_{k+1}-48 u_{k}-51 u_{k-1}\right]+\frac{1}{2 h^{2}}\left[39 u_{k+1}^{\prime}+96 u_{k}^{\prime}+15 u_{k-1}^{\prime}\right] \\
& =u_{k+1}^{\prime \prime \prime}-\frac{h^{3}}{10} u_{k}^{v i}+O\left(h^{4}\right) .
\end{aligned}
$$

Similarly,

$$
\begin{aligned}
\overline{\bar{u}}_{k-1}^{\prime \prime \prime} & =\frac{1}{2 h^{3}}\left[99 u_{k-1}-48 u_{k}-51 u_{k+1}\right]+\frac{1}{2 h^{2}}\left[39 u_{k-1}^{\prime}+96 u_{k}^{\prime}+15 u_{k+1}^{\prime}\right] \\
& =u_{k-1}^{\prime \prime \prime}+\frac{h^{3}}{10} u_{k}^{v i}+O\left(h^{4}\right)
\end{aligned}
$$

Let

$$
\alpha_{k}=\frac{\partial f}{\partial u_{k}^{\prime \prime}}, \quad \beta_{k}=\frac{\partial f}{\partial u_{k}^{\prime \prime \prime}}
$$


From (3.5a), (3.5b), (3.6), and (2.9a) it follows that $\bar{f}_{k \pm 1}$ provides the $O\left(h^{2}\right)$ approximation for $f_{k \pm 1}$ and

$$
\bar{f}_{k \pm 1}=f_{k \pm 1}-\frac{h^{2}}{3} u_{k}^{i v} \alpha_{k}-\frac{2 h^{2}}{5} u_{k}^{v} \beta_{k} \pm O\left(h^{3}\right) .
$$

Also, from (2.9b), (3.11), (3.14), and (3.15) we obtain the $O\left(h^{3}\right)$ approximation for $f_{k \pm 1}$ :

$$
\overline{\bar{f}}_{k \pm 1}=f_{k \pm 1} \mp \frac{h^{3}}{15} u_{k}^{v} \alpha_{k} \mp \frac{h^{3}}{10} u_{k}^{v i} \beta_{k}+O\left(h^{4}\right) \text {. }
$$

From (2.9c), (3.9), and (3.10) it follows that $\overline{\bar{f}}_{k}$ provides an $O\left(h^{4}\right)$ approximation for $f_{k}$

$$
\overline{\bar{f}}_{k}=f_{k}+O\left(h^{4}\right)
$$

From (3.18) and (3.19) we get

$$
\overline{\bar{f}}_{k+1}+\overline{\bar{f}}_{k-1}+13 \overline{\bar{f}}_{k}=f_{k+1}+f_{k-1}+13 f_{k}+O\left(h^{4}\right) .
$$

With the help of (3.2) and (3.20), from (2.10), we obtain that the local truncation error associated with the difference scheme (2.10) is of $O\left(h^{8}\right)$. Similarly, we can verify that the local truncation error associated with the difference scheme (2.11) is of $O\left(h^{7}\right)$.

Thus, we have the following result.

Theorem 3.1. Consider the fourth-order nonlinear ordinary differential equation (1.1) along with the boundary conditions (1.2). Let $u \in C^{8}[a, b]$, and the function $f$ is sufficiently differentiable with respect to its arguments. Then, the difference methods (2.10) and (2.11) with the approximations of $u^{\prime \prime}$ and $u^{\prime \prime \prime}$ listed in (2.3)-(2.8) are of $O\left(h^{4}\right)$.

If the differential (1.1) is linear, then the difference method (2.10) and (2.11) in the matrix form can be written as

$$
\left[\begin{array}{ll}
\mathbf{A}_{11} & \mathbf{A}_{12} \\
\mathbf{A}_{21} & \mathbf{A}_{22}
\end{array}\right]\left[\begin{array}{l}
\mathbf{u} \\
\mathbf{u}^{\prime}
\end{array}\right]=\left[\begin{array}{l}
\mathbf{d}_{1} \\
\mathbf{d}_{2}
\end{array}\right],
$$

where $\mathbf{A}_{11}, \mathbf{A}_{12}, \mathbf{A}_{21}$, and $\mathbf{A}_{22}$ are the $N$ th-order tri-diagonal matrices and $\mathbf{d}_{1}$ and $\mathbf{d}_{2}$ are vectors consisting of right-hand side functions and some boundary conditions associated with the block system.

The block successive over relaxation (BSOR) method (See Mohanty and Evans [26, 28]) is given by

$$
\begin{gathered}
\mathbf{A}_{11} \mathbf{u}^{(n+1)}=\omega\left[-\mathbf{A}_{12}\left(\mathbf{u}^{\prime}\right)^{(n)}+\mathbf{d}_{1}\right]+(1-\omega) \mathbf{A}_{11} \mathbf{u}^{(n)}, \quad n=0,1,2, \ldots, \\
\mathbf{A}_{22}\left(\mathbf{u}^{\prime}\right)^{(n+1)}=\omega\left[-\mathbf{A}_{21} \mathbf{u}^{(n+1)}+\mathbf{d}_{2}\right]+(1-\omega) \mathbf{A}_{22}\left(\mathbf{u}^{\prime}\right)^{(n)}, \quad n=0,1,2, \ldots,
\end{gathered}
$$


where $\omega$ is a parameter known as relaxation parameter. With $\omega=1$, the BSOR method reduces to block Gauss-Seidel (BGS) method. If $\omega>1$ or $\omega<1$, we have overrelaxation or underrelaxation, respectively.

If $f\left(x, u(x), v(x), u^{\prime}(x), v^{\prime}(x)\right)$ is nonlinear, the difference equations (2.2a), (2.2b) or (2.10), (2.11) form a coupled nonlinear system. To solve the coupled nonlinear system we apply the Newton NBSOR method.

We first write the difference equations (2.2a), (2.2b) or (2.10), (2.11) as

$$
\begin{aligned}
& \boldsymbol{\Phi}(\mathbf{u}, \mathbf{v})=0, \\
& \Psi(\mathbf{u}, \mathbf{v})=0,
\end{aligned}
$$

where

$$
\mathbf{u}=\left[\begin{array}{c}
u_{1} \\
u_{2} \\
\vdots \\
u_{N}
\end{array}\right], \quad \mathbf{v}=\left[\begin{array}{c}
v_{1} \\
v_{2} \\
\vdots \\
v_{N}
\end{array}\right], \quad \boldsymbol{\Phi}(\mathbf{u}, \mathbf{v})=\left[\begin{array}{c}
\phi_{1}(\mathbf{u}, \mathbf{v}) \\
\phi_{2}(\mathbf{u}, \mathbf{v}) \\
\vdots \\
\phi_{N}(\mathbf{u}, \mathbf{v})
\end{array}\right], \quad \Psi(\mathbf{u}, \mathbf{v})=\left[\begin{array}{c}
\psi_{1}(\mathbf{u}, \mathbf{v}) \\
\psi_{2}(\mathbf{u}, \mathbf{v}) \\
\vdots \\
\psi_{N}(\mathbf{u}, \mathbf{v})
\end{array}\right]
$$

Let

$$
\mathbf{J}=\left[\begin{array}{ll}
\mathbf{T}_{11} & \mathbf{T}_{12} \\
\mathbf{T}_{21} & \mathbf{T}_{22}
\end{array}\right]
$$

be the Jacobian of $\boldsymbol{\Phi}$ and $\Psi$, which is the $2 N$ th-order block tridiagonal matrix, where

$$
\begin{aligned}
& \mathbf{T}_{11}=\frac{\partial\left(\phi_{1}, \phi_{2}, \ldots, \phi_{N}\right)}{\partial\left(u_{1}, u_{2}, \ldots, u_{N}\right)}=\left[\begin{array}{cccc}
\frac{\partial \phi_{1}}{\partial u_{1}} & \frac{\partial \phi_{1}}{\partial u_{2}} & & \\
\frac{\partial \phi_{2}}{\partial u_{1}} & \frac{\partial \phi_{2}}{\partial u_{2}} & \frac{\partial \phi_{2}}{\partial u_{3}} & 0 \\
& 0 & \frac{\partial \phi_{N}}{\partial u_{N-1}} & \frac{\partial \phi_{N}}{\partial u_{N}}
\end{array}\right] \\
& \mathbf{T}_{12}=\frac{\partial\left(\phi_{1}, \phi_{2}, \ldots, \phi_{N}\right)}{\partial\left(v_{1}, v_{2}, \ldots, v_{N}\right)}
\end{aligned}
$$


Advances in Numerical Analysis

$$
\begin{aligned}
& \mathbf{T}_{21}=\frac{\partial\left(\psi_{1}, \psi_{2}, \ldots, \psi_{N}\right)}{\partial\left(u_{1}, u_{2}, \ldots, u_{N}\right)} \\
& \mathbf{T}_{22}=\frac{\partial\left(\psi_{1}, \psi_{2}, \ldots, \psi_{N}\right)}{\partial\left(v_{1}, v_{2}, \ldots, v_{N}\right)}
\end{aligned}
$$

are the $N$ th-order tridiagonal matrices.

In the NBSOR method starting with any initial approximation $\left(\mathbf{u}^{(0)}, \mathbf{v}^{(0)}\right)$ of $\left(\mathbf{u}^{(k)}, \mathbf{v}^{(k)}\right)$, $k=1(1) N$, we define

$$
\begin{aligned}
& \mathbf{u}^{(n+1)}=\mathbf{u}^{(n)}+\Delta \mathbf{u}^{(n)}, \quad n=0,1,2, \ldots, \\
& \mathbf{v}^{(n+1)}=\mathbf{v}^{(n)}+\Delta \mathbf{v}^{(n)}, \quad n=0,1,2, \ldots,
\end{aligned}
$$

where $\Delta \mathbf{u}$ and $\Delta \mathbf{v}$ are intermediate values obtained by solving the matrix equation for NBSOR method given by

$$
\left[\begin{array}{ll}
\mathbf{T}_{11} & \mathbf{T}_{12} \\
\mathbf{T}_{21} & \mathbf{T}_{22}
\end{array}\right]\left[\begin{array}{l}
\Delta \mathbf{u} \\
\Delta \mathbf{v}
\end{array}\right]=\left[\begin{array}{l}
-\boldsymbol{\Phi} \\
-\boldsymbol{\Psi}
\end{array}\right]
$$

The above system can be solved for $\Delta \mathbf{u}^{(n)}$ and $\Delta \mathbf{v}^{(n)}$ by using the block SOR method (inner iterative method) as follows:

$$
\begin{gathered}
\mathbf{T}_{11} \Delta \mathbf{u}^{(n+1)}=\omega\left[-\mathbf{\Phi}\left(\mathbf{u}^{(n)}, \mathbf{v}^{(n)}\right)-\mathbf{T}_{12} \Delta \mathbf{v}^{(n)}\right]+(1-\omega) \mathbf{T}_{11} \Delta \mathbf{u}^{(n)}, \quad n=0,1,2, \ldots, \\
\mathbf{T}_{22} \Delta \mathbf{v}^{(n+1)}=\omega\left[-\Psi\left(\mathbf{u}^{(n)}, \mathbf{v}^{(n)}\right)-\mathbf{T}_{21} \Delta \mathbf{u}^{(n+1)}\right]+(1-\omega) \mathbf{T}_{22} \Delta \mathbf{v}^{(n)}, \quad n=0,1,2, \ldots,
\end{gathered}
$$

where $\omega \in(0,2)$ is a relaxation parameter and $n=0,1,2, \ldots$. The above system of equations can be solved by using the tridiagonal solver. In order for this method to converge it is sufficient that the initial approximation $\left(\mathbf{u}^{(0)}, \mathbf{v}^{(0)}\right)$ be close to the solution.

\section{Convergence and Stability Analysis}

Consider the model problem $u^{i v}=f(x)$, where $f$ is a function of $x$ only. Applying the fourthorder difference methods (2.10) and (2.11) to the above equation, we get

$$
\begin{gathered}
\left(u_{k-1}-2 u_{k}+u_{k+1}\right)+\frac{h}{2}\left(v_{k-1}-v_{k+1}\right)=\frac{-h^{4}}{180}\left(f_{k+1}+f_{k-1}+13 f_{k}\right), \\
\frac{3}{h}\left(u_{k-1}-u_{k+1}\right)+\left(v_{k-1}+4 v_{k}+v_{k+1}\right)=\frac{h^{3}}{60}\left(f_{k+1}-f_{k-1}\right)
\end{gathered}
$$

where we denote $u^{\prime}=v$. 
Let us denote by $\mathbf{P}=[1,0,1], \mathbf{L}=[1,1,1]$, and $\mathbf{M}=[1,0,-1]$ the $N$ th-order tridiagonal matrices.

The system of (4.1) can be written in the block form as

$$
\left[\begin{array}{cc}
(\mathbf{L}-3 \mathbf{I}) & \frac{\mathrm{h}}{2} \mathbf{M} \\
\frac{3}{\mathbf{h}} \mathbf{M} & (\mathbf{L}+3 \mathbf{I})
\end{array}\right]\left[\begin{array}{l}
\mathbf{u} \\
\mathbf{v}
\end{array}\right]=\left[\begin{array}{l}
\mathbf{d}_{1} \\
\mathbf{d}_{2}
\end{array}\right]
$$

where $\mathbf{u}$ and $\mathbf{v}$ are two $\mathbf{N}$-dimensional solution vectors and $\mathbf{d}_{1}, \mathbf{d}_{2}$ are vectors consisting of right-hand side functions and some boundary values associated with (4.1). The BSOR method for the scheme is

$$
\begin{aligned}
\mathbf{u}^{(n+1)} & =(1-\omega) \mathbf{u}^{(n)}-\frac{\omega h}{2}(\mathbf{L}-3 \mathbf{I})^{-1} \mathbf{M}(\mathbf{v})^{(n)}+\omega(\mathbf{L}-3 \mathbf{I})^{-1} \mathbf{d}_{1} \\
(\mathbf{v})^{(n+1)} & =(1-\omega)(\mathbf{v})^{(n)}-\frac{3 \omega}{h}(\mathbf{L}+3 \mathbf{I})^{-1} \mathbf{M} \mathbf{u}^{(n+1)}+\omega(\mathbf{L}+3 \mathbf{I})^{-1} \mathbf{d}_{2}
\end{aligned}
$$

The associated block SOR and block Jacobi iteration matrices are given by

$$
\begin{gathered}
\mathbf{L}_{\omega}=\left[\begin{array}{cr}
(1-\omega) \mathbf{I} & \frac{-\omega h}{2}(\mathbf{L}-3 \mathbf{I})^{-1} \mathbf{M} \\
\frac{-3 \omega}{h}(\mathbf{L}+3 \mathbf{I})^{-1} \mathbf{M} & (1-\omega) \mathbf{I}
\end{array}\right], \\
\mathbf{B}=\left[\begin{array}{cc}
0 & \frac{-h}{2}(\mathbf{L}-3 \mathbf{I})^{-1} \mathbf{M} \\
\frac{-3}{h}(\mathbf{L}+3 \mathbf{I})^{-1} \mathbf{M} & 0
\end{array}\right],
\end{gathered}
$$

and $\lambda$ and $\eta$ are the eigenvalues associated with the corresponding matrices $\mathbf{L}_{\omega}$ and $\mathbf{B}$, which are related by the equation

$$
(\lambda+\omega-1)^{2}=\lambda \omega^{2} \eta^{2}
$$

Let $\left[\begin{array}{l}\mathbf{v}_{1} \\ \mathbf{v}_{2}\end{array}\right]$ be the eigenvector associated with the eigenvalue $\eta$ so that

$$
\left[\begin{array}{cc}
0 & \frac{-h}{2}(\mathbf{L}-3 \mathbf{I})^{-1} \mathbf{M} \\
\frac{-3}{h}(\mathbf{L}+3 \mathbf{I})^{-1} \mathbf{M} & 0
\end{array}\right]\left[\begin{array}{l}
\mathbf{v}_{1} \\
\mathbf{v}_{2}
\end{array}\right]=\left[\begin{array}{l}
\mathbf{v}_{1} \\
\mathbf{v}_{2}
\end{array}\right] \eta
$$

That is,

$$
\begin{aligned}
& \frac{-h}{2}(\mathbf{L}-3 \mathbf{I})^{-1} \mathbf{M} \mathbf{v}_{2}=\eta \mathbf{v}_{1}, \\
& \frac{-3}{h}(\mathbf{L}+3 \mathbf{I})^{-1} \mathbf{M} \mathbf{v}_{1}=\eta \mathbf{v}_{2} .
\end{aligned}
$$


On eliminating $\mathbf{v}_{2}$, we obtain

$$
\frac{3}{2}(\mathbf{L}-3 \mathbf{I})^{-1} \mathbf{M}(\mathbf{L}+3 \mathbf{I})^{-1} M \mathbf{v}_{1}=\eta^{2} \mathbf{v}_{1}
$$

The rate of convergence of the BSOR method is given by $-\log \left(\rho\left(\mathbf{L}_{\omega}\right)\right)$.

The rate of convergence of the BSOR method is dependent on the eigenvalues of B (through relation (4.5)), which are given by $(3 \tau / 2)=\eta^{2}$, where $\tau$ are the eigenvalues of $(\mathbf{L}-3 \mathbf{I})^{-1} \mathbf{M}(\mathbf{L}+3 \mathbf{I})^{-1} \mathbf{M}$.

Hence, we can determine the optimal parameter as

$$
\omega_{0}=\frac{2}{1+\sqrt{1-(3 / 2) \bar{\tau}}}
$$

where $\bar{\tau}=S\left((\mathbf{L}-3 \mathbf{I})^{-1} \mathbf{M}(\mathbf{L}+3 \mathbf{I})^{-1} \mathbf{M}\right)$.

Thus, we can determine the convergence factor

$$
\bar{\lambda}=\omega_{0}-1=\frac{1-\sqrt{1-(3 / 2) \bar{\tau}}}{1+\sqrt{1-(3 / 2) \bar{\tau}}} .
$$

For convergence, we must have $|\bar{\lambda}|<1$ to give the range

$$
0<\bar{\tau}<\frac{2}{3}
$$

Hence, we get the convergence.

Thus, we have the following result.

Theorem 4.1. The iteration method of the form (4.3) for the solution of $u^{i v}=f(x)$ converges if $0<\bar{\tau}<2 / 3$, where $\bar{\tau}=S\left((\mathbf{L}-3 \mathbf{I})^{-1} \mathbf{M}(\mathbf{L}+3 \mathbf{I})^{-1} \mathbf{M}\right), \mathbf{L}=[1,1,1]$, and $\mathbf{M}=[1,0,-1]$ are the $N \times N$ tridiagonal matrices.

Now, we discuss the stability analysis.

An iterative method for (4.1) can be written as

$$
\begin{aligned}
\mathbf{u}^{(k+1)} & =\frac{1}{2} \mathbf{P} \mathbf{u}^{(k)}+\frac{h}{4} \mathbf{M} \mathbf{v}^{(k)}+\mathbf{R H U}, \\
\mathbf{v}^{(k+1)} & =\frac{-3}{4 h} \mathbf{M} \mathbf{u}^{(k)}-\frac{1}{4} \mathbf{P} \mathbf{v}^{(k)}+\mathbf{R H V},
\end{aligned}
$$

where $\mathbf{u}^{(k)}, \mathbf{v}^{(k)}$ are solution vectors at the $k$ th iteration and RHU, RHV are right-hand side vectors consisting of boundary and homogenous function values.

The above iterative method in matrix form can be written as

$$
\left[\begin{array}{l}
\mathbf{u}^{(k+1)} \\
\mathbf{v}^{(k+1)}
\end{array}\right]=\mathbf{G}\left[\begin{array}{l}
\mathbf{u}^{(k)} \\
\mathbf{v}^{(k)}
\end{array}\right]+\mathbf{R H}
$$


where

$$
\mathbf{G}=\left[\begin{array}{cc}
\frac{1}{2} \mathbf{P} & \frac{h}{4} \mathbf{M} \\
\frac{-3}{4 h} \mathbf{M} & \frac{-1}{4} \mathbf{P}
\end{array}\right], \quad \mathbf{R H}=\left[\begin{array}{l}
\mathbf{R H U} \\
\mathbf{R H V}
\end{array}\right]
$$

The eigenvalues of $\mathbf{P}$ and $\mathbf{M}$ are $2 \cos k \pi /(N+1)$ and $2 i \cos k \pi /(N+1)$, respectively, where $k=1,2, \ldots, N$. The characteristic equation of the matrix $\mathrm{G}$ is given by

$$
\operatorname{det}\left[\begin{array}{cc}
\frac{1}{2} \mathbf{P}-\xi \mathbf{I}_{N} & \frac{h}{4} \mathbf{M} \\
\frac{-3}{4 h} \mathbf{M} & \frac{-1}{4} \mathbf{P}-\xi \mathbf{I}_{N}
\end{array}\right]=0
$$

Thus the eigenvalues of $\mathbf{G}$ are given by

$$
\operatorname{det}\left[\frac{-1}{4} \mathbf{P}-\xi \mathbf{I}_{N}\right] \times \operatorname{det}\left[\left(\frac{1}{2} \mathbf{P}-\xi \mathbf{I}_{N}\right)+\frac{3}{16} \mathbf{M}\left(\frac{-1}{4} \mathbf{P}-\xi \mathbf{I}_{N}\right)^{-1} \mathbf{M}\right]=0 \text {. }
$$

The proposed iterative method (4.13) is stable as long as the maximum absolute eigenvalues of the iteration matrix are less than or equal to one. It has been verified computationally that all eigenvalues of the system (4.16) are less than one. Hence, the iterative method (4.1) is stable.

\section{Application to Singular Equation}

Consider a singular fourth-order linear ordinary differential equation of the form

$$
\Delta^{4} u \equiv\left(\frac{d^{2}}{d r^{2}}+\frac{1}{r} \frac{d}{d r}\right)^{2} u=f(r), \quad 0<r<1,
$$

or equivalently

$$
u^{i v}=b(r) u^{\prime \prime \prime}+c(r) u^{\prime \prime}+d(r) u^{\prime}+f(r), \quad 0<r<1,
$$

where

$$
b(r)=-2 / r, \quad c(r)=1 / r^{2}, \quad d(r)=-1 / r^{3} .
$$

The above equation represents fourth-order ordinary differential equation in cylindrical polar coordinates.

The boundary conditions are given by

$$
u(0)=A_{0}, \quad u^{\prime}(0)=A_{1}, \quad u(1)=B_{0}, \quad u^{\prime}(1)=B_{1},
$$

where $A_{0}, A_{1}, B_{0}$, and $B_{1}$ are constants. 
Applying the difference scheme (2.2a), (2.2b) to the singular equation (5.2), we obtain a second-order difference method

$$
\begin{gathered}
-2\left(u_{k+1}-2 u_{k}+u_{k-1}\right)+h\left(u_{k+1}^{\prime}-u_{k-1}^{\prime}\right)=\frac{h^{4}}{6}\left(b_{k} \bar{u}_{k}^{\prime \prime \prime}+c_{k} \bar{u}_{k}^{\prime \prime}+d_{k} u_{k}^{\prime}+f_{k}\right), \\
-3\left(u_{k+1}-u_{k-1}\right)+h\left(u_{k+1}^{\prime}+4 u_{k}^{\prime}+u_{k-1}^{\prime}\right)=0, \quad k=1(1) N .
\end{gathered}
$$

Applying the fourth-order difference scheme (2.10) to the singular equation (5.2), we obtain

$$
\begin{aligned}
-2\left(u_{k+1}-\right. & \left.2 u_{k}+u_{k-1}\right)+h\left(u_{k+1}^{\prime}-u_{k-1}^{\prime}\right) \\
=\frac{h^{4}}{90}[ & \left(b_{k+1} \overline{\bar{u}}_{k+1}^{\prime \prime \prime}+c_{k+1} \overline{\bar{u}}_{k+1}^{\prime \prime}+d_{k+1} u_{k+1}^{\prime}+f_{k+1}\right) \\
& +\left(b_{k-1} \overline{\bar{u}}_{k-1}^{\prime \prime \prime}+c_{k-1} \overline{\bar{u}}_{k-1}^{\prime \prime}+d_{k-1} u_{k-1}^{\prime}+f_{k-1}\right) \\
& \left.+\left(13 b_{k} \overline{\bar{u}}_{k}^{\prime \prime \prime}+13 c_{k} \overline{\bar{u}}_{k}^{\prime \prime}+13 d_{k} u_{k}^{\prime}+13 f_{k}\right)\right], \quad k=1(1) N,
\end{aligned}
$$

where

$$
b_{k \pm 1}=b\left(r_{k \pm 1}\right), \quad c_{k \pm 1}=c\left(r_{k \pm 1}\right), \quad d_{k \pm 1}=d\left(r_{k \pm 1}\right), \quad f_{k \pm 1}=f\left(r_{k \pm 1}\right) .
$$

Note that the scheme fails when the solution is to be determined at $k=1$. We overcome the difficulty by modifying the method in such a way that the solutions retain the order and accuracy even in the vicinity of the singularity $r=0$ (see [29]). We consider the following approximations:

$$
\begin{aligned}
& b_{k \pm 1}=b_{k} \pm h b_{k}^{\prime}+\frac{h^{2}}{2 !} b_{k}^{\prime \prime}+O\left( \pm h^{3}\right), \\
& c_{k \pm 1}=c_{k} \pm h c_{k}^{\prime}+\frac{h^{2}}{2 !} c_{k}^{\prime \prime}+O\left( \pm h^{3}\right), \\
& d_{k \pm 1}=d_{k} \pm h d_{k}^{\prime}+\frac{h^{2}}{2 !} d_{k}^{\prime \prime}+O\left( \pm h^{3}\right), \\
& f_{k \pm 1}=f_{k} \pm h f_{k}^{\prime}+\frac{h^{2}}{2 !} f_{k}^{\prime \prime}+O\left( \pm h^{3}\right) .
\end{aligned}
$$

Using the approximation (5.8) in (5.6) and neglecting higher-order terms, we can rewrite (5.6) in compact operator form as

$$
\begin{array}{r}
-2\left(u_{k+1}-2 u_{k}+u_{k-1}\right)+h\left(u_{k+1}^{\prime}-u_{k-1}^{\prime}\right) \\
=\frac{h^{2}}{90}\left(-24 b_{k}^{\prime}+18 c_{k}-4 h^{2} c_{k}^{\prime \prime}\right) \delta_{x}^{2} u_{k}
\end{array}
$$




$$
\begin{aligned}
& +\frac{h}{180}\left(45 b_{k}-75 h^{2} b_{k}^{\prime \prime}-6 h^{2} c_{k}^{\prime}\right)\left(2 \mu_{x} \delta_{x}\right) u_{k} \\
& +\frac{h^{4}}{90}\left(\frac{-45 b_{k}}{h^{2}}+75 b_{k}^{\prime \prime}+6 c_{k}^{\prime}+15 d_{k}+h^{2} d_{k}^{\prime \prime}\right) u_{k}^{\prime} \\
& +\frac{h^{2}}{180}\left(15 b_{k}+27 h^{2} b_{k}^{\prime \prime}+6 h^{2} c_{k}^{\prime}+2 h^{2} d_{k}\right)\left(2 \mu_{x} \delta_{x}\right) u_{k}^{\prime} \\
& +\frac{h^{3}}{180}\left(24 b_{k}^{\prime}-3 c_{k}+5 h^{2} c_{k}^{\prime \prime}+2 h^{2} d_{k}^{\prime}\right)\left(2 \mu_{x} \delta_{x}\right) u_{k} \\
& +\frac{h^{4}}{90}\left(15 f_{k}+h^{2} f_{k}^{\prime \prime}\right), \quad k=1(1) N .
\end{aligned}
$$

Similarly, using the difference scheme (2.11), a fourth-order approximation for the derivative $u^{\prime}$ for the singular equation (5.2) in the compact form may be written as

$$
\begin{aligned}
-3 & \left(u_{k+1}-u_{k-1}\right)+h\left(u_{k+1}^{\prime}+4 u_{k}^{\prime}+u_{k-1}^{\prime}\right) \\
= & \frac{h}{60}\left(-24 b_{k}\right) \delta_{x}^{2} u_{k}+\frac{h^{2}}{60}\left(-3 b_{k}^{\prime}\right)\left(2 \mu_{x} \delta_{x}\right) u_{k}+\frac{h^{3}}{60}\left(2 c_{k}+3 b_{k}^{\prime}\right) \delta_{x}^{2} u_{k}^{\prime} \\
& +\frac{h^{2}}{60}\left(12 b_{k}+h^{2}\left(c_{k}^{\prime}+d_{k}\right)\right)\left(2 \mu_{x} \delta_{x}\right) u_{k}^{\prime}+\frac{h^{3}}{60}\left(6 b_{k}^{\prime}+2 h^{2} d_{k}^{\prime}\right) u_{k}^{\prime}+\frac{h^{4}}{60}\left(2 h f_{k}^{\prime}\right), \quad k=1(1) N,
\end{aligned}
$$

where $\delta_{r} u_{k}=u_{k+1 / 2}-u_{k-1 / 2}, \mu_{r} u_{k}=\left(u_{k+1 / 2}+u_{k-1 / 2}\right) / 2$.

Finite difference equations (5.9) and (5.10) along with the boundary conditions (5.4) gives a $2 N \times 2 N$ linear system of equations for the unknowns $u_{1}, u_{2}, \ldots, u_{N}, u_{1}^{\prime}, u_{2}^{\prime}, \ldots, u_{N}^{\prime}$. The resulting block tri-diagonal system can be solved using the BGS method. The schemes (5.9) and (5.10) are free from the terms $1 /(k \pm 1)$, hence very easily solved for $k=1(1) N$ in the region $(0,1)$.

Consider the coupled nonlinear singular equations

$$
\begin{gathered}
u^{I V}=a(r)\left[u^{\prime} v^{\prime \prime}+v^{\prime} u^{\prime \prime}\right]+f(r), \quad 0<r<1, \\
v^{I V}=-a(r) u^{\prime} u^{\prime \prime}+g(r), \quad 0<r<1,
\end{gathered}
$$

where $a(r)=1 / r$, with known boundary conditions $u(0), v(0), u^{\prime}(0), v^{\prime}(0), u(1), v(1)$, $u^{\prime}(1)$, and $v^{\prime}(1)$. The coupled equations represent model equations of equilibrium for a load symmetrical about the centre (see [30]).

The second-order difference scheme for solving the system (5.11) is given by

$$
\begin{gathered}
-2\left(u_{k+1}-2 u_{k}+u_{k-1}\right)+h\left(u_{k+1}^{\prime}-u_{k-1}^{\prime}\right)=\frac{h^{4}}{6}\left[a_{k}\left(u_{k}^{\prime} \bar{v}_{k}^{\prime \prime}+v_{k}^{\prime} \bar{u}_{k}^{\prime \prime}\right)+f_{k}\right], \\
-2\left(v_{k+1}-2 v_{k}+v_{k-1}\right)+h\left(v_{k+1}^{\prime}-v_{k-1}^{\prime}\right)=\frac{h^{4}}{6}\left[-a_{k} u_{k}^{\prime} \bar{u}_{k}^{\prime \prime}+g_{k}\right],
\end{gathered}
$$


Advances in Numerical Analysis

$$
\begin{aligned}
& -3\left(u_{k+1}-u_{k-1}\right)+h\left(u_{k+1}^{\prime}+4 u_{k}^{\prime}+u_{k-1}^{\prime}\right)=0, \\
& -3\left(v_{k+1}-v_{k-1}\right)+h\left(v_{k+1}^{\prime}+4 v_{k}^{\prime}+v_{k-1}^{\prime}\right)=0,
\end{aligned}
$$

where $a_{k}=a\left(r_{k}\right), f_{k}=f\left(r_{k}\right)$, and $g_{k}=g\left(r_{k}\right)$. given by

The fourth-order difference scheme for solving the system (5.11) for $u, v, u^{\prime}$, and $v^{\prime}$ is

$$
\begin{aligned}
& -2\left(u_{k+1}-2 u_{k}+u_{k-1}\right)+h\left(u_{k+1}^{\prime}-u_{k-1}^{\prime}\right) \\
& =H_{1}\left[15 f_{k}+h^{2} f_{k}^{\prime \prime}\right] \\
& +H_{1} a_{11}\left[u_{k+1}^{\prime}\left\{\frac{-1}{2 h^{2}}\left(11 v_{k+1}-16 v_{k}+5 v_{k-1}\right)+\frac{1}{h}\left(4 v_{k+1}^{\prime}-v_{k-1}^{\prime}\right)\right\}\right. \\
& \left.+v_{k+1}^{\prime}\left\{\frac{-1}{2 h^{2}}\left(11 u_{k+1}-16 u_{k}+5 u_{k-1}\right)+\frac{1}{h}\left(4 u_{k+1}^{\prime}-u_{k-1}^{\prime}\right)\right\}\right] \\
& +H_{1} a_{12}\left[u_{k-1}^{\prime}\left\{\frac{-1}{2 h^{2}}\left(11 v_{k-1}-16 v_{k}+5 v_{k+1}\right)-\frac{1}{h}\left(4 v_{k-1}^{\prime}-v_{k+1}^{\prime}\right)\right\}\right. \\
& +H_{1} a_{10}\left[u_{k-1}^{\prime}\left\{\frac{-1}{2 h^{2}}\left(11 u_{k-1}-16 u_{k}+5 u_{k+1}\right)-\frac{1}{h}\left(4 u_{k-1}^{\prime}-u_{k+1}^{\prime}\right)\right\}\right] \\
& \left.\quad+v_{k}^{\prime}\left\{\frac{2}{h^{2}}\left(u_{k+1}-2 u_{k}+u_{k-1}\right)-\frac{1}{2 h}\left(u_{k+1}^{\prime}-u_{k-1}^{\prime}\right)\right\}\right] \\
&
\end{aligned}
$$




$$
\begin{aligned}
& -3\left(v_{k+1}-v_{k-1}\right)+h\left(v_{k+1}^{\prime}+4 v_{k}^{\prime}+v_{k-1}^{\prime}\right) \\
= & H_{2}\left(2 h g_{k}^{\prime}\right)-H_{2} a_{11} u_{k+1}^{\prime}\left\{\frac{3 u_{k+1}^{\prime}-4 u_{k}^{\prime}+u_{k-1}^{\prime}}{2 h}\right\}-H_{2} a_{12} u_{k-1}^{\prime}\left\{\frac{-3 u_{k-1}^{\prime}+4 u_{k}^{\prime}-u_{k+1}^{\prime}}{2 h}\right\},
\end{aligned}
$$

where

$$
\begin{gathered}
a_{k}=a\left(r_{k}\right), \quad f_{k}=f\left(r_{k}\right), \quad g_{k}=g\left(r_{k}\right), \\
a_{11}=a_{k}+h a_{k}^{\prime}+\frac{h^{2}}{2} a_{k}^{\prime \prime}, \quad a_{12}=a_{k}-h a_{k}^{\prime}+\frac{h^{2}}{2} a_{k}^{\prime \prime}, \quad a_{10}=a_{k} .
\end{gathered}
$$

The scheme (5.13) is free from the terms $1 / k \pm 1$, hence very easily solved for $k=1(1) N$ in the region $(0,1)$. The system $(5.13)$ can be solved using the NBSOR method.

Consider the boundary value problem [31]

$$
\begin{gathered}
y^{i v}-\lambda y y^{\prime \prime}=f(x), \\
y(0)=V_{0}, \quad y(1)=V_{1}, \quad y^{\prime}(0)=0, \quad y^{\prime}(1)=0 .
\end{gathered}
$$

This arises from the time-dependent Navier-Strokes equations for axisymmetric flow of an incompressible fluid contained between infinite disks that occupy the planes $z=-d$ and $z=d$. The disks are porous and the fluid is injected or extracted normally with velocity $V_{0}$ at $z=-d$ and $V_{1}$ at $z=d$. Here, $=d / v$, where $v$ is the kinematic viscosity.

\section{Numerical Illustrations}

To illustrate our method and to demonstrate computationally its convergence, we have solved the following linear problem using the BGS method (See [25, 32-35]), whose exact solution is known to us. We have taken $[0,1]$ as our region of integration. The right-hand side functions and the boundary conditions are obtained using the exact solution. The iterations were stopped when the absolute error tolerance became $\leq 10^{-12}$. All computations were performed using double length arithmetic.

Problem 1. The problem is to solve (5.2) subject to the boundary conditions (5.4). The exact solution is $u=r^{4} \sin r$. The root mean square errors (RMSEs) are tabulated in Table 1. The graph of the errors for $N=32$ is given in Figure 1 .

Problem 2. The boundary value problem is to solve (5.15). The exact solution is $\left(1-x^{2}\right) \exp (x)$. The maximum absolute errors (MAE) and RMSE are tabulated in Table 2.

Problem 3. The system of nonlinear equation (5.11) is to be solved subject to the natural boundary conditions. The exact solutions are $u=\cos (r)$ and $v=\exp (r)$. The MAE and RMSE are tabulated in Table 3. 


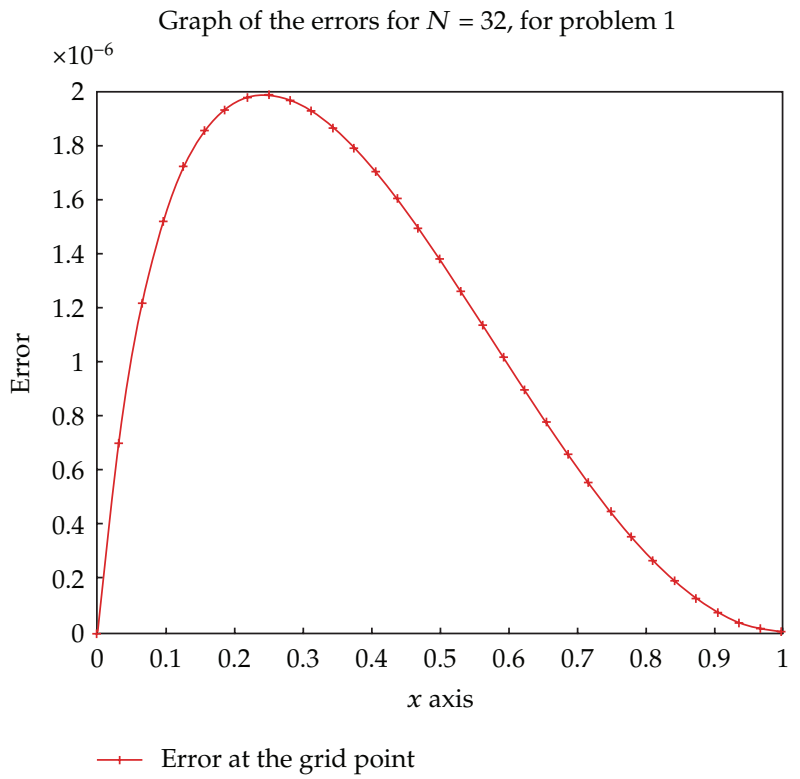

Figure 1

Table 1: The RMSE.

\begin{tabular}{lllccc}
\hline & & \multicolumn{2}{c}{ 2nd-order method } & \multicolumn{2}{c}{ 4th-order method } \\
\multicolumn{1}{l}{$1 / 8$} & $u$ & $0.9512(-03)$ & $0.6737(-03)$ & $0.6018(-03)$ & $0.4089(-03)$ \\
& $u^{\prime}$ & $0.3162(-02)$ & $0.2137(-02)$ & $0.2961(-02)$ & $0.1402(-02)$ \\
\hline \multirow{2}{*}{$1 / 16$} & $u$ & $0.2212(-03)$ & $0.1501(-03)$ & $0.3799(-04)$ & $0.2502(-04)$ \\
& $u^{\prime}$ & $0.7021(-03)$ & $0.4995(-03)$ & $0.3022(-03)$ & $0.9846(-04)$ \\
\multirow{2}{*}{$1 / 32$} & $u$ & $0.5410(-04)$ & $0.3576(-04)$ & $0.1989(-05)$ & $0.1304(-05)$ \\
& $u^{\prime}$ & $0.1729(-03)$ & $0.1206(-03)$ & $0.2302(-05)$ & $0.5629(-05)$ \\
\multirow{2}{*}{$1 / 64$} & $u$ & $0.1339(-04)$ & $0.8769(-05)$ & $0.9996(-07)$ & $0.6578(-07)$ \\
& $u^{\prime}$ & $0.4298(-04)$ & $0.2972(-04)$ & $0.1562(-05)$ & $0.2983(-06)$ \\
\hline \multirow{2}{*}{$1 / 128$} & $u$ & $0.3363(-05)$ & $0.2174(-05)$ & $0.4198(-08)$ & $0.3345(-08)$ \\
& $u^{\prime}$ & $0.1072(-04)$ & $0.7383(-05)$ & $0.9892(-07)$ & $0.1538(-07)$ \\
\hline
\end{tabular}

Observe that for the linear singular problem for $\alpha=1, h_{1}=1 / 64, h_{2}=1 / 128$, $\log \left(e_{h_{1}} / e_{h_{2}}\right) / \log \left(h_{1} / h_{2}\right)=\log (0.6578(-07) / 0.3345(-08)) / \log 2 \approx 4$. Thus, we obtain fourthorder convergence for $u$. Similarly, we get fourth-order convergence for $u^{\prime}$.

\section{Concluding Remarks}

The numerical results confirm that the proposed finite difference methods yield second- and fourth-order convergence for the solution and its derivative for the fourth-order ordinary differential equation. Difference formulas for mesh points near the boundary are obtained without using the fictitious points. The proposed method is applicable to problems in polar coordinates and the derivative of the solution is obtained as the by-product of the method. We 
Table 2: The MAE and RMSE.

\begin{tabular}{|c|c|c|c|c|c|}
\hline \multirow[b]{2}{*}{$h$} & & \multicolumn{2}{|c|}{ 2nd-order method } & \multicolumn{2}{|c|}{ 4th-order method } \\
\hline & & MAE & RMSE & MAE & RMSE \\
\hline \multicolumn{6}{|c|}{$\lambda=1.0$} \\
\hline \multirow{2}{*}{$1 / 8$} & $u$ & $0.8435(-04)$ & $0.5652(-04)$ & $0.2744(-05)$ & $0.1880(-05)$ \\
\hline & $u^{\prime}$ & $0.3552(-03)$ & $0.2322(-03)$ & $0.1058(-04)$ & $0.7178(-05)$ \\
\hline \multirow{2}{*}{$1 / 16$} & $u$ & $0.2292(-04)$ & $0.1499(-04)$ & $0.1854(-06)$ & $0.1217(-06)$ \\
\hline & $u^{\prime}$ & $0.7762(-04)$ & $0.5391(-04)$ & $0.6695(-06)$ & $0.4351(-06)$ \\
\hline \multirow{2}{*}{$1 / 32$} & $u$ & $0.5844(-05)$ & $0.3771(-05)$ & $0.1151(-07)$ & $0.7446(-08)$ \\
\hline & $u^{\prime}$ & $0.1885(-04)$ & $0.1322(-04)$ & $0.4115(-07)$ & $0.2618(-07)$ \\
\hline \multicolumn{6}{|c|}{$\lambda=100$} \\
\hline \multirow{2}{*}{$1 / 8$} & $u$ & $0.1961(-02)$ & $0.1401(-02)$ & $0.5662(-04)$ & $0.3877(-04)$ \\
\hline & $u^{\prime}$ & $0.6514(-02)$ & $0.4687(-02)$ & $0.3824(-03)$ & $0.1910(-03)$ \\
\hline \multirow{2}{*}{$1 / 16$} & $u$ & $0.4808(-03)$ & $0.3312(-03)$ & $0.3821(-05)$ & $0.2558(-05)$ \\
\hline & $u^{\prime}$ & $0.1597(-02)$ & $0.1110(-02)$ & $0.1929(-04)$ & $0.9922(-05)$ \\
\hline \multirow{2}{*}{$1 / 32$} & $u$ & $0.1197(-03)$ & $0.8103(-04)$ & $0.2435(-06)$ & $0.1609(-06)$ \\
\hline & $u^{\prime}$ & $0.4023(-03)$ & $0.2714(-03)$ & $0.1135(-05)$ & $0.5843(-06)$ \\
\hline
\end{tabular}

Table 3: The MAE and RMSE.

\begin{tabular}{|c|c|c|c|c|c|}
\hline \multirow[b]{2}{*}{$h$} & & \multicolumn{2}{|c|}{ 2nd-order method } & \multicolumn{2}{|c|}{ 4th-order method } \\
\hline & & MAE & RMSE & MAE & RMSE \\
\hline \multirow{4}{*}{$1 / 10$} & $u$ & $0.7141(-05)$ & $0.4859(-05)$ & $0.2673(-05)$ & $0.1818(-05)$ \\
\hline & $v$ & $0.3339(-05)$ & $0.2240(-05)$ & $0.6974(-06)$ & $0.4745(-06)$ \\
\hline & $u^{\prime}$ & $0.2488(-04)$ & $0.1683(-04)$ & $0.1266(-04)$ & $0.6538(-05)$ \\
\hline & $v^{\prime}$ & $0.1126(-04)$ & $0.8137(-05)$ & $0.2548(-05)$ & $0.1656(-05)$ \\
\hline \multirow{4}{*}{$1 / 20$} & $u$ & $0.1806(-05)$ & $0.1182(-05)$ & $0.2467(-06)$ & $0.1639(-06)$ \\
\hline & $v$ & $0.8550(-06)$ & $0.5595(-06)$ & $0.4846(-07)$ & $0.3212(-07)$ \\
\hline & $u^{\prime}$ & $0.6486(-05)$ & $0.4128(-05)$ & $0.1167(-05)$ & $0.5946(-06)$ \\
\hline & $v^{\prime}$ & $0.2659(-05)$ & $0.1957(-05)$ & $0.1795(-06)$ & $0.1119(-06)$ \\
\hline \multirow{4}{*}{$1 / 40$} & $u$ & $0.4514(-06)$ & $0.2917(-06)$ & $0.2048(-07)$ & $0.1346(-07)$ \\
\hline & $v$ & $0.2152(-06)$ & $0.1391(-06)$ & $0.3315(-08)$ & $0.2161(-08)$ \\
\hline & $u^{\prime}$ & $0.1620(-05)$ & $0.1020(-05)$ & $0.1027(-06)$ & $0.4911(-07)$ \\
\hline & $v^{\prime}$ & $0.6757(-06)$ & $0.4828(-06)$ & $0.1222(-07)$ & $0.7515(-08)$ \\
\hline \multirow{4}{*}{$1 / 80$} & $u$ & $0.1125(-06)$ & $0.7216(-07)$ & $0.1510(-08)$ & $0.9836(-09)$ \\
\hline & $v$ & $0.5307(-07)$ & $0.3442(-07)$ & $0.2931(-09)$ & $0.1883(-09)$ \\
\hline & $u^{\prime}$ & $0.4038(-06)$ & $0.2525(-06)$ & $0.8190(-08)$ & $0.3662(-08)$ \\
\hline & $v^{\prime}$ & $0.1686(-06)$ & $0.1192(-06)$ & $0.9925(-09)$ & $0.6537(-09)$ \\
\hline \multirow{4}{*}{$1 / 160$} & $u$ & $0.3050(-07)$ & $0.1946(-07)$ & $0.1213(-09)$ & $0.7945(-10)$ \\
\hline & $v$ & $0.1461(-07)$ & $0.9331(-08)$ & $0.1358(-10)$ & $0.8769(-11)$ \\
\hline & $u^{\prime}$ & $0.1076(-06)$ & $0.6812(-07)$ & $0.6420(-09)$ & $0.2913(-09)$ \\
\hline & $v^{\prime}$ & $0.4591(-07)$ & $0.3237(-07)$ & $0.5009(-10)$ & $0.3048(-10)$ \\
\hline
\end{tabular}

employ the BGS method to solve the block matrix systems of the linear singular problem and the BSOR method to solve the nonlinear singular problem. We have solved here a physical problem that arises in the axisymmetric flow of an incompressible fluid. 


\section{Acknowledgments}

This research was supported by "The Council of Scientific and Industrial Research" under Research Grant no. 09/045(0836)2009-EMR-I. The authors thank the reviewers for their valuable suggestions, which substantially improved the standard of the paper.

\section{References}

[1] S. P. Timoshenko, Theory of Elastic Stability, McGraw-Hill Book, New York, NY, USA, 2nd edition, 1961.

[2] A. R. Aftabizadeh, "Existence and uniqueness theorems for fourth-order boundary value problems," Journal of Mathematical Analysis and Applications, vol. 116, no. 2, pp. 415-426, 1986.

[3] R. P. Agarwal and P. R. Krishnamoorthy, "Boundary value problems for $n$th order ordinary differential equations," Bulletin of the Institute of Mathematics. Academia Sinica, vol. 7, no. 2, pp. 211-230, 1979.

[4] R. P. Agarwal, "Boundary value problems for higher order differential equations," Bulletin of the Institute of Mathematics. Academia Sinica, vol. 9, no. 1, pp. 47-61, 1981.

[5] R. P. Agarwal and G. Akrivis, "Boundary value problems occurring in plate deflection theory," Journal of Computational and Applied Mathematics, vol. 8, no. 3, pp. 145-154, 1982.

[6] Z. Bai, B. Huang, and W. Ge, "The iterative solutions for some fourth-order $p$-Laplace equation boundary value problems," Applied Mathematics Letters, vol. 19, no. 1, pp. 8-14, 2006.

[7] J. R. Graef and L. Kong, "A necessary and sufficient condition for existence of positive solutions of nonlinear boundary value problems," Nonlinear Analysis, vol. 66, no. 11, pp. 2389-2412, 2007.

[8] J. R. Graef, C. Qian, and B. Yang, "A three point boundary value problem for nonlinear fourth order differential equations," Journal of Mathematical Analysis and Applications, vol. 287, no. 1, pp. 217-233, 2003.

[9] M. D. Greenberg, Differential Equations and Linear Algebra, chapter 7, Prentice Hall, Engelwood Cliffs, NJ, USA, 2001.

[10] R. K. Mohanty, "A fourth-order finite difference method for the general one-dimensional nonlinear biharmonic problems of first kind," Journal of Computational and Applied Mathematics, vol. 114, no. 2, pp. 275-290, 2000.

[11] R. K. Nagle and E. B. Saff, Fundamentals of Differential Equations, chapter 6, The Benjamin/Cummingspp, 1986.

[12] M. A. Noor and S. T. Mohyud-Din, "An efficient method for fourth-order boundary value problems," Computers \& Mathematics with Applications, vol. 54, no. 7-8, pp. 1101-1111, 2007.

[13] D. O'Regan, "Solvability of some fourth (and higher) order singular boundary value problems," Journal of Mathematical Analysis and Applications, vol. 161, no. 1, pp. 78-116, 1991.

[14] J. Schröder, "Numerical error bounds for fourth order boundary value problems, simultaneous estimation of $u(x)$ and $u^{\prime \prime}(x), "$ Numerische Mathematik, vol. 44, no. 2, pp. 233-245, 1984.

[15] V. Shanthi and N. Ramanujam, "A numerical method for boundary value problems for singularly perturbed fourth-order ordinary differential equations," Applied Mathematics and Computation, vol. 129, no. 2-3, pp. 269-294, 2002.

[16] D. G. Zill and M. R. Cullen, Differential Equations with Boundary-Value Problems, chapter 5, Brooks Cole, NewYork, NY, USA, 1997.

[17] R. A. Usmani, "Finite difference methods for computing eigenvalues of fourth order boundary value problems," International Journal of Mathematics and Mathematical Sciences, vol. 9, no. 1, pp. 137-143, 1986.

[18] R. A. Usmani and M. Sakai, "Two new finite difference methods for computing eigenvalues of a fourth order linear boundary value problem," International Journal of Mathematics and Mathematical Sciences, vol. 10, no. 3, pp. 525-530, 1987.

[19] R. A. Usmani and P. J. Taylor, "Finite difference methods for solving $\left[p(x) y^{\prime \prime}\right]$ " $+q(x) y=r(x)$," International Journal of Computer Mathematics, vol. 14, no. 3-4, pp. 277-293, 1983.

[20] E. H. Twizell and S. I. A. Tirmizi, "Multiderivative methods for linear fourth order boundary value problems," Tech. Rep. TR/06/84, Department of Mathematics And Statistics, Brunel University, 1984.

[21] R. P. Agarwal and Y. M. Chow, "Iterative methods for a fourth order boundary value problem," Journal of Computational and Applied Mathematics, vol. 10, no. 2, pp. 203-217, 1984. 
[22] A. Cabada, "The method of lower and upper solutions for second, third, fourth, and higher order boundary value problems," Journal of Mathematical Analysis and Applications, vol. 185, no. 2, pp. 302320, 1994.

[23] D. Franco, D. O’Regan, and J. Perán, "Fourth-order problems with nonlinear boundary conditions," Journal of Computational and Applied Mathematics, vol. 174, no. 2, pp. 315-327, 2005.

[24] G. Han and F. Li, "Multiple solutions of some fourth-order boundary value problems," Nonlinear Analysis, vol. 66, no. 11, pp. 2591-2603, 2007.

[25] L. A. Hageman and D. M. Young, Applied Iterative Methods, Dover Publications, Mineola, NY, USA, 2004.

[26] R. K. Mohanty and D. J. Evans, “Block iterative methods for one-dimensional nonlinear biharmonic problems on a parallel computer," Parallel Algorithms and Applications, vol. 13, no. 3, pp. 239-263, 1999.

[27] M. M. Chawla, "A fourth-order tridiagonal finite difference method for general non-linear two-point boundary value problems with mixed boundary conditions," Journal of the Institute of Mathematics and its Applications, vol. 21, no. 1, pp. 83-93, 1978.

[28] R. K. Mohanty and D. J. Evans, "New algorithms for the numerical solution of one dimensional singular biharmonic problems of second kind," International Journal of Computer Mathematics, vol. 73, no. 1, pp. 105-124, 1999.

[29] D. J. Evans and R. K. Mohanty, "Alternating group explicit method for the numerical solution of nonlinear singular two-point boundary value problems using a fourth order finite difference method," International Journal of Computer Mathematics, vol. 79, no. 10, pp. 1121-1133, 2002.

[30] J. Prescott, Applied Elasticity, Dover Publications Inc, New York, NY, USA, 1961.

[31] A. R. Elcrat, "On the radial flow of a viscous fluid between porous disks," Archive for Rational Mechanics and Analysis, vol. 61, no. 1, pp. 91-96, 1976.

[32] C. T. Kelly, Iterative Methods for Linear and Non-Linear Equations, SIAM Publication, Philadelphia, Pa, USA, 1995.

[33] Y. Saad, Iterative Methods for Sparse Linear Systems, Society for Industrial and Applied Mathematics, Philadelphia, Pa, USA, 2nd edition, 2003.

[34] R. S. Varga, Matrix Iterative Analysis, vol. 27 of Springer Series in Computational Mathematics, Springer, Berlin, Germany, 2000.

[35] D. M. Young, Iterative Solution of Large Linear Systems, Dover Publications, Mineola, NY, USA, 2003. 


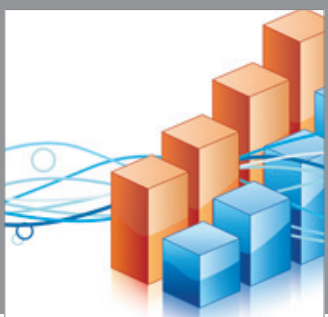

Advances in

Operations Research

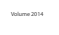

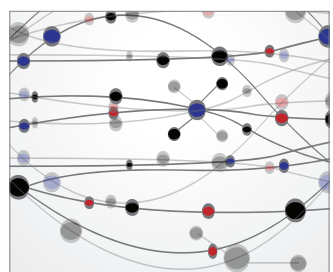

\section{The Scientific} World Journal
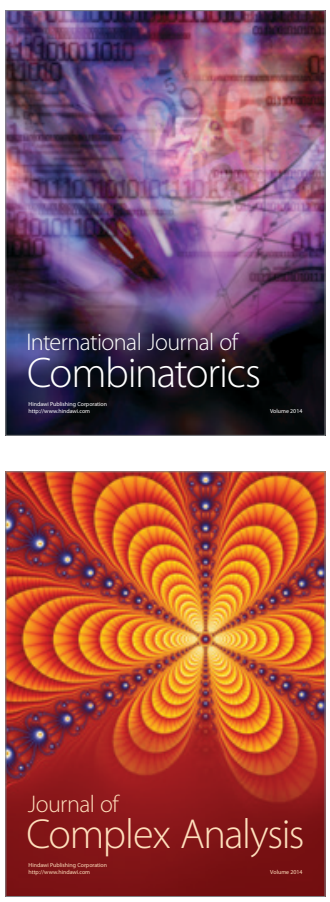

International Journal of

Mathematics and

Mathematical

Sciences
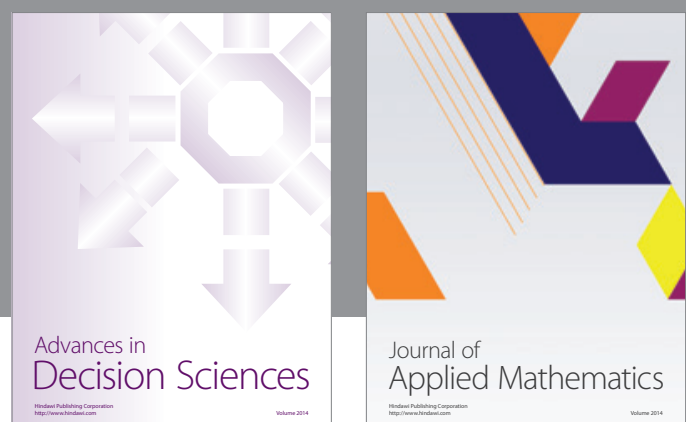

Journal of

Applied Mathematics
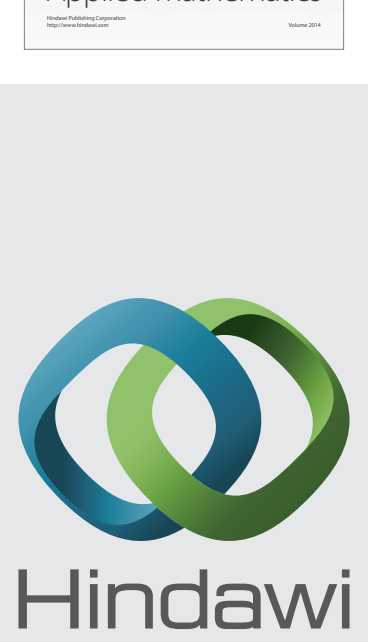

Submit your manuscripts at http://www.hindawi.com
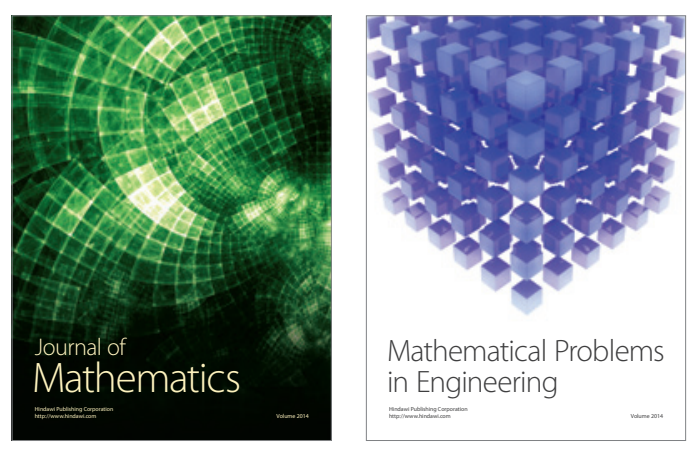

Mathematical Problems in Engineering
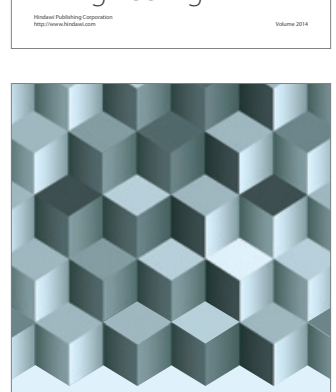

Journal of

Function Spaces
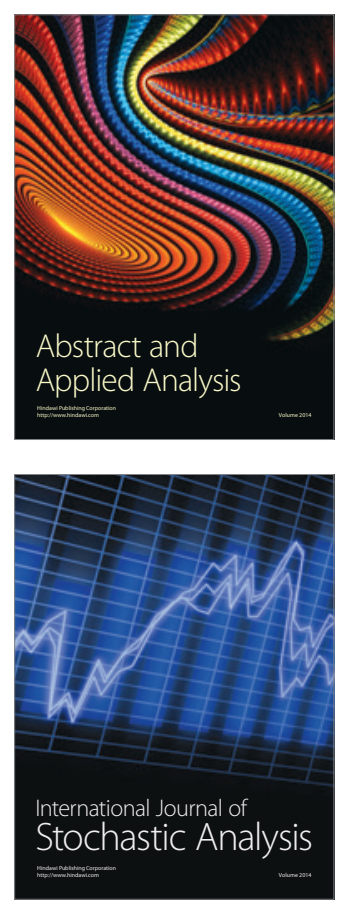

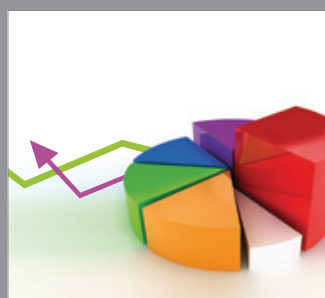

ournal of

Probability and Statistics

Promensencen
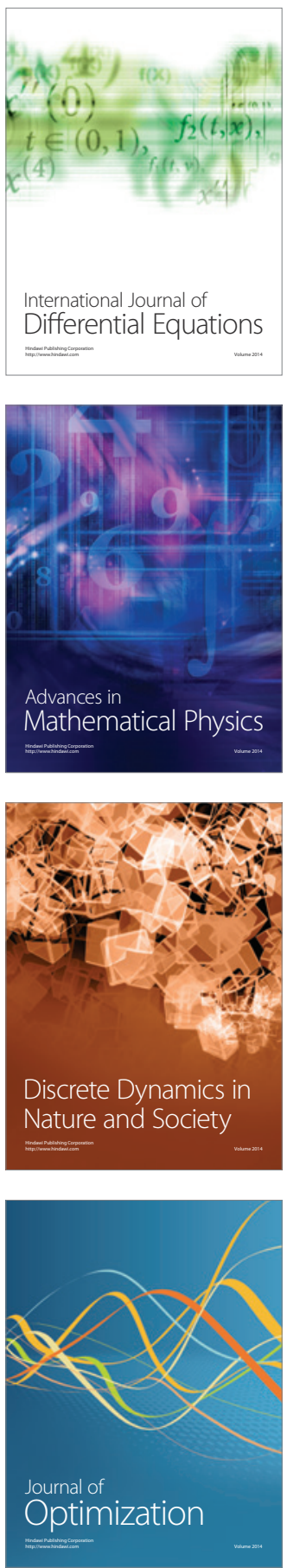